\title{
Dietary patterns are associated with insulin resistance in Chinese adults without known diabetes
}

\author{
Hui Zuo ${ }^{1,2 *}$, Zumin Shi ${ }^{1,3}$, Baojun Yuan ${ }^{1}$, Yue Dai ${ }^{1}$, Xiaoqun Pan ${ }^{4}$, Gaolin Wu ${ }^{1}$ and Akhtar Hussain ${ }^{2}$ \\ ${ }^{1}$ Department of Nutrition and Food Hygiene, Jiangsu Provincial Center for Disease Control and Prevention, \\ 172 Jiangsu Road, Nanjing 210009, People's Republic of China \\ ${ }^{2}$ Department of General Practice and Community Medicine, Institute of Health and Society, Faculty of Medicine, \\ University of Oslo, Oslo, Norway \\ ${ }^{3}$ Discipline of Medicine, University of Adelaide, Adelaide, SA, Australia \\ ${ }^{4}$ Department of Non-communicable Disease Control, Jiangsu Provincial Center for Disease Control and Prevention, Nanjing, \\ People's Republic of China
}

(Submitted 29 March 2012 - Final revision received 19 July 2012 - Accepted 19 July 2012 - First published online 19 September 2012)

\begin{abstract}
The aim of the present study was to examine the association between dietary patterns and insulin resistance in Chinese adults without known diabetes. Study subjects were 1070 Chinese adults aged 18 years and above in Jiangsu Province who participated in the 2006 wave of the China Health and Nutrition Survey. Usual dietary intake was assessed by using a validated FFQ. Dietary patterns were identified by factor analysis using a principal component analysis method. Insulin resistance was defined as the highest quartile of the homeostasis model assessment of insulin resistance (HOMA-IR) scores. We derived four dietary patterns in our population by factor analysis: the Western, High-wheat, Traditional and Hedonic pattern. After adjusted for potential confounders, the Western pattern was significantly associated with greater odds for insulin resistance $(P$ for trend $=0.009)$, while a significant negative association was found between the Hedonic pattern and insulin resistance $(P$ for trend $=0.035$. Compared with the lowest quartile of the Western pattern, the highest quartile had higher odds of insulin resistance (adjusted OR 1·89, $95 \%$ CI 1·12, 3.19). There was a $42 \%$ decrease in the odds after adjustment for all covariates in the highest quartile of the Hedonic pattern, compared with the lowest quartile (adjusted OR 0.58, 95\% CI 0.34, 0.99). HOMA-IR levels as a continuous variable also increased across the quartiles of the Western pattern and decreased across the quartiles of the Hedonic pattern. In conclusion, dietary patterns were significantly associated with insulin resistance in Chinese adults without known diabetes.
\end{abstract}

Key words: Dietary patterns: Insulin resistance: Factor analysis: Adults: China

Insulin resistance is a pathophysiological condition where the insulin receptor is less sensitive and therefore insulin in a natural dose is not enough to produce normal biological effects. It is recognised as the underlying cause of many adverse health events such as type 2 diabetes and the metabolic syndrome ${ }^{(1)}$, and also it often occurs before the events. Cumulative studies have revealed that diet plays an important role in insulin resistance. Studies ${ }^{(2-6)}$ on the association between the intake of individual dietary components and insulin resistance have been reported. For instance, vegetables and fruit ${ }^{(2)}$, low-fat dairy products ${ }^{(3)}$ and regular alcohol consumption ${ }^{(4,5)}$ were found to be inversely associated with insulin resistance, while dietary SFA intake ${ }^{(6)}$ has been reported to be positively associated with insulin resistance.
The traditional approach focusing on a single food or nutrient has its methodological and conceptual limitations ${ }^{(7,8)}$. It fails to take into account nutrient interaction and to detect small effects from single nutrients. More importantly in reality, no one consumes just one single food or nutrient. It cannot provide tangible dietary advice as well ${ }^{(8)}$. In nutritional epidemiology, dietary pattern analysis has emerged as an alternative method to assess dietary exposures. It has been shown to be a useful method to evaluate diet-disease relationships ${ }^{(7-9)}$. Due to its ability to examine the effects of overall diet, dietary pattern analysis has been often used to identify the relationship between diet and insulin resistance/diabetes ${ }^{(10-13)}$.

China has seen the fastest economic growth in the world in the context of its transition from a planned economy to a market economy. The economic development has

Abbreviations: CHNS, China Health and Nutrition Survey; HOMA-IR, homeostasis model assessment of insulin resistance. 
a substantial effect on nutritional and lifestyle changes, which further contribute to increasing rates of obesity and diabetes in the population ${ }^{(14)}$. Few studies have shown that dietary patterns were associated with diabetes and glucose tolerance abnormality in China ${ }^{(11,12)}$ or among the Chinese population $^{(13)}$. Identifying the association between dietary patterns and insulin resistance would help prevent those health events at an earlier stage. However, no study in China can be found on such an association. The aim of the present study was to examine the association between dietary patterns identified by factor analysis and insulin resistance in Chinese adults without known diabetes.

\section{Subjects and methods \\ Subjects and study design}

Study subjects were the participants in the 2006 wave of the China Health and Nutrition Survey (CHNS) in Jiangsu Province. This study is a nationwide ongoing open cohort designed to evaluate the effects of health, nutrition and family planning policies on population health and nutritional status under economic transformation in China. More detailed information has been described elsewhere ${ }^{(15)}$. Jiangsu was the only province that collected blood samples in the CHNS project in 2006. The study sample was from six areas (Suzhou, Yangzhou, Shuyang, Taixing, Haimen and Jinhu) by a multistage random cluster process. In total, sixteen villages and townships within the counties and eight urban and suburban neighbourhoods within the cities were selected randomly. The present study was conducted according to the guidelines laid down in the Declaration of Helsinki and all procedures involving human subjects were approved by the review board in the Jiangsu Provincial Center for Disease Control and Prevention. All participants provided written informed consent, with a response rate of $91 \cdot 3 \%$.

For the present study, 1156 subjects aged 18 years and above with dietary survey and blood sample information were included. To improve the validity of the data, forty-six individuals were further excluded due to their implausible daily energy intake $(<2092 \mathrm{~kJ} / \mathrm{d}(<500 \mathrm{kcal} / \mathrm{d}),>16736 \mathrm{~kJ} / \mathrm{d}$ ( $>4000 \mathrm{kcal} / \mathrm{d})$ for women, $>17573 \mathrm{~kJ} / \mathrm{d}(>4200 \mathrm{kcal} / \mathrm{d})$ for men) based on the Chinese context ${ }^{(16)}$. Also, forty persons who had previously diagnosed diabetes were excluded, leaving 1070 participants (488 men and 582 women) for the present analysis. Among them, there were sixty-two previously undiagnosed cases with diabetes.

\section{Assessment of dietary intake}

A validated semi-quantitative FFQ was used to collect dietary intake information by a face-to-face interview, which was only used in Jiangsu in the 2006 CHNS $^{(17,18)}$. Participants were asked to recall their usual frequency and quantity of intakes of thirty-three food groups and beverages during the previous year with a series of detailed questions. Intake of each food item was calculated by multiplying the reported frequency of the food by estimated portion size of the food per time. Intakes of foods were converted into $\mathrm{g} / \mathrm{d}$ for further analysis. Total energy and nutrients were computed by using the Chinese Food Composition Table ${ }^{(19)}$.

\section{Dietary patterns}

Dietary patterns were identified by factor analysis using a principal component analysis method. Cheese was excluded due to no consumption among the present study subjects. Several food groups and beverages in the FFQ were aggregated into main food groups, mainly according to macronutrient composition and culinary use, i.e. tofu was a proportional aggregation of tofu, dried bean curd and soyabeans according to the protein content; pickled vegetables were the total of preserved vegetables, pickled vegetables, kimchi and Chinese sauerkraut; beverages represented juice and other soft drinks excluding water. As a result, twenty-six foods/food groups were entered into the analysis in absolute weight in grams.

The PROC FACTOR procedure in SAS (SAS Institute) was used to perform the analysis. The number of factors retained was determined by eigenvalue $(>1.25)$, scree plot, factor interpretability and the variance explained $(>5 \%)$ by each factor. The factors retained were then rotated with an orthogonal rotation ('Varimax' option in SAS) to improve interpretability and minimise the correlation between the factors ${ }^{(9)}$. From these analyses, a four-factor solution was selected. A factor score was then calculated for each subject for each of the four factors, as the sum of the products of the factor loading coefficient and standardised daily intake of each food/food group associated with that pattern. Labelling of the factors was primarily descriptive and based on our interpretation of the pattern structures.

\section{Laboratory measurements}

Venous blood samples were obtained in the morning after an overnight fast of at least $8-12 \mathrm{~h}$. The fasting status was verbally confirmed by subjects before the blood sampling. All blood samples were collected in three vacuum tubes and processed within $3 \mathrm{~h}$. Serum was stored at $-70^{\circ} \mathrm{C}$ until laboratory assays. Fasting glucose was assessed by an enzymology method using the OLYMPUS Chemistry Analyzer AU400 (Mishima Olympus Company Limited). Plasma insulin was measured by an ELISA Kit (Millipore Corporation). The homeostasis model assessment of insulin resistance (HOMA-IR) score was calculated as ${ }^{(20)}$ :

$$
\begin{aligned}
\text { HOMA }= & \text { IR-fasting insulin }(\mathrm{mU} / \mathrm{l}) \\
& \times \text { fasting glucose }(\mathrm{mmol} / \mathrm{l}) / 22.5 .
\end{aligned}
$$

Its highest quartile was used to define insulin resistance, as published previously ${ }^{(21,22)}$

\section{Covariates}

Anthropometric data were measured directly by trained health workers following standard protocols. Weight in light clothing 
and without shoes was measured to the nearest $0 \cdot 1 \mathrm{~kg}$ and height was measured to the nearest $0 \cdot 1 \mathrm{~cm}$. BMI was calculated as weight in $\mathrm{kg}$ over the square of height in $\mathrm{m}$. We additionally added BMI into the logistic regression model to examine whether the association was mediated by obesity.

Income was estimated by family annual income per capita. It was categorised as low (<5000 Yuan), medium (5000-10000 Yuan) and high ( $>10000$ Yuan). Physical activities including domestic, occupational, transportation and leisure-time physical activity were collected by staff-administered questionnaires. They were assessed in terms of metabolic equivalents $\times \mathrm{h}$ /week to account for both intensity and time spent on activities. The level of physical activity was the product of time spent in each activity multiplied by specific metabolic equivalent values based on the 'Compendium of Physical Activities, ${ }^{, 23,24)}$. Other demographic and lifestyle covariates, such as sex, age and smoking status, were collected by a questionnaire-based interview. Current smoking status was coded as dichotomous variables (yes/no).

\section{Statistical analysis}

Factor scores for each pattern were categorised into quartiles (quartiles 1 and 4 represented low and high adherence, respectively, to each dietary pattern). Sample characteristics are presented as mean values and standard deviations for continuous variables, and percentages for categorical variables. Fasting glucose, insulin and HOMA-IR are expressed as medians (interquartile ranges) due to skewness and normalised by log transformation before fitting into linear regression models.

Dietary fibre, micronutrients, alcohol, beer and wine intakes were adjusted for sex, age and total energy intake. Linear trend was tested by the general linear model for continuous variables and the $\chi^{2}$ test for trend for categorical variables. Multivariate logistic regression was used to determine the association between food patterns and insulin resistance, adjusted for sociodemographic and lifestyle factors including sex, age, income, energy intake, physical activity and smoking status. In order to make full use of available information, HOMA-IR levels as a continuous variable across the quartiles of dietary patterns were also computed and tested for linear trend by the general linear model. All the analyses were performed using SAS software (version 8.1; SAS Institute). Statistical significance was considered when $P<0.05$ (two-sided).

\section{Results}

We identified four major dietary patterns by factor analysis. Factor loadings for the four dietary patterns are shown in Table 1 . The four dietary patterns were labelled as 'Western' (characterised by animal foods, milk, cake, etc.), 'Highwheat' (high in wheat instead of rice, whole grain and beef/ lamb), 'Traditional' (high in eggs, tofu, organ meat, pickled vegetables, etc.) and 'Hedonic' (high in beer, wine and alcohols, and fresh vegetables). In total, the four factors explained $29.8 \%$ of the variance in dietary intake $(9 \cdot 4,8 \cdot 5,6.4$ and $5.5 \%$, respectively).

Table 1. Rotated factor loading matrix for the four dietary patterns among 1070 Chinese adults*

\begin{tabular}{|c|c|c|c|c|}
\hline Foods/food groups & Western & High-wheat & Traditional & Hedonic \\
\hline Beer & - & - & - & 0.60 \\
\hline Wine & - & - & - & 0.41 \\
\hline Low-degree alcohol† & - & - & - & 0.39 \\
\hline Tubers & 0.29 & - & - & 0.31 \\
\hline Fresh vegetables & - & -0.43 & - & 0.29 \\
\hline Fried wheat & 0.44 & - & - & 0.28 \\
\hline Beverages & 0.38 & - & -0.22 & 0.28 \\
\hline Pork & 0.51 & - & - & 0.27 \\
\hline High-degree alcohol & - & - & 0.22 & 0.27 \\
\hline Beef/lamb & 0.27 & 0.24 & - & - \\
\hline Organ meat & 0.23 & - & 0.40 & - \\
\hline Poultry & 0.44 & - & 0.45 & - \\
\hline Soyabean milk & 0.49 & - & - & - \\
\hline Aquatic products & 0.37 & -0.32 & 0.49 & - \\
\hline Whole grains & - & 0.75 & - & - \\
\hline Pickled vegetables & - & - & 0.33 & - \\
\hline Rice & - & -0.68 & - & - \\
\hline Wheat & - & 0.75 & - & - \\
\hline Tofu & - & - & 0.52 & - \\
\hline Eggs & - & - & 0.55 & - \\
\hline Nuts & - & - & $0 \cdot 24$ & - \\
\hline Milk powder & - & - & $0 \cdot 21$ & - \\
\hline Fruits & 0.50 & - & 0.22 & - \\
\hline Cake & 0.50 & - & - & -0.21 \\
\hline Milk & 0.52 & - & - & -0.24 \\
\hline Yogurt & 0.44 & - & - & - \\
\hline
\end{tabular}

* Absolute values $<0.20$ are indicated by a dash for simplicity

† Alcohol content by volume $\leq 38 \%$.

$\ddagger$ Alcohol content by volume $>38 \%$. 
Table 2. Characteristics and dietary intakes of the study participants for the lowest (Q1) and highest (Q4) quartiles of the four dietary patterns for 1070 adults in the China Health and Nutrition Survey study

(Mean values and standard deviations or standard errors)

\begin{tabular}{|c|c|c|c|c|c|c|c|c|c|c|c|c|c|c|c|c|c|c|c|c|}
\hline & \multicolumn{5}{|c|}{ Western } & \multicolumn{5}{|c|}{ High-wheat } & \multicolumn{5}{|c|}{ Traditional } & \multicolumn{5}{|c|}{ Hedonic } \\
\hline & \multicolumn{2}{|c|}{ Q1 } & \multicolumn{2}{|c|}{ Q4 } & \multirow[b]{2}{*}{$P^{*}$} & \multicolumn{2}{|c|}{ Q1 } & \multicolumn{2}{|c|}{ Q4 } & \multirow[b]{2}{*}{$P^{*}$} & \multicolumn{2}{|c|}{ Q1 } & \multicolumn{2}{|c|}{ Q4 } & \multirow[b]{2}{*}{$P^{*}$} & \multicolumn{2}{|c|}{ Q1 } & \multicolumn{2}{|c|}{ Q4 } & \multirow[b]{2}{*}{$P^{\star}$} \\
\hline & Mean & SD & Mean & SD & & Mean & SD & Mean & SD & & Mean & SD & Mean & SD & & Mean & SD & Mean & SD & \\
\hline Age (years) & \multirow{2}{*}{\multicolumn{2}{|c|}{$\begin{array}{l}49 \cdot 1 \\
42 \cdot 4 \\
\end{array}$}} & \multirow{2}{*}{\multicolumn{2}{|c|}{47.9}} & 0.006 & 49.5 & 13.8 & 47.5 & 13.5 & 0.025 & 49.2 & $15 \cdot 6$ & 49.9 & 13.6 & 0.800 & 52.6 & $16 \cdot 0$ & $47 \cdot 0$ & $12 \cdot 7$ & $<0.001$ \\
\hline Men (\%) & & & & & 0.869 & \multicolumn{2}{|c|}{50.2} & 41 & & 0.032 & 37 & & 50 & & 0.003 & 32 & & 74 & & $<0.001$ \\
\hline $\mathrm{BMI}\left(\mathrm{kg} / \mathrm{m}^{2}\right)$ & 23.0 & 3.5 & 23.6 & 3.4 & 0.098 & $23 \cdot 2$ & 3.5 & 23.6 & 3.6 & 0.663 & $22 \cdot 8$ & 3.8 & 23.8 & 3.5 & 0.009 & 24.0 & 4.3 & 23.4 & 3.5 & 0.023 \\
\hline Smoking (\%) & 29 & & 24 & 9 & 0.524 & 28 & & 24 . & & 0.220 & 19 & & 33 & & $<0.001$ & 14 & & 45 & & $<0.001$ \\
\hline $\begin{array}{l}\text { Physical activity } \\
\text { (MET } \times \text { h/week) }\end{array}$ & 214.5 & $125 \cdot 1$ & $123 \cdot 2$ & 87.9 & $<0.001$ & $196 \cdot 2$ & 128.4 & $166 \cdot 3$ & 95.2 & $<0.001$ & $163 \cdot 8$ & $116 \cdot 8$ & $171 \cdot 8$ & 123.7 & 0.768 & $140 \cdot 6$ & $117 \cdot 8$ & $186 \cdot 9$ & 121.9 & $<0.001$ \\
\hline Energy/nutrient intake & & & & & & & & & & & & & & & & & & & & \\
\hline Total energy & & & & & & & & & & & & & & & & & & & & \\
\hline $\mathrm{kcal} / \mathrm{d}$ & 2443 & 772 & 2500 & 623 & $<0.001$ & 2618 & 573 & 2576 & 678 & $<0.001$ & 1821 & 673 & 2745 & 634 & $<0.001$ & 1909 & 641 & 2803 & 673 & $<0.001$ \\
\hline $\mathrm{kJ} / \mathrm{d}$ & 10220 & 3229 & 10459 & 2608 & $<0.001$ & 10956 & 2399 & 10777 & 2838 & $<0.001$ & 7622 & 2816 & 11486 & 2651 & $<0.001$ & 7989 & 2680 & 11729 & 2817 & $<0.001$ \\
\hline Carbohydrate (g/d) & 393.9 & $142 \cdot 3$ & 349.1 & 102.5 & $<0.001$ & 409.9 & 97.5 & $410 \cdot 0$ & 133.1 & $<0.001$ & 281.4 & $125 \cdot 3$ & 387.4 & 113.5 & $<0.001$ & 286.9 & $111 \cdot 3$ & 403.7 & 124.5 & $<0.001$ \\
\hline Protein $(\mathrm{g} / \mathrm{d})$ & 67.5 & $27 \cdot 6$ & $81 \cdot 0$ & 21.4 & $<0.001$ & 73.6 & 18.5 & 80.6 & $25 \cdot 6$ & $<0.001$ & $50 \cdot 1$ & $20 \cdot 0$ & $90 \cdot 2$ & $22 \cdot 9$ & $<0.001$ & 58.8 & 23.3 & 82.5 & 23.6 & $<0.001$ \\
\hline Fat $(\mathrm{g} / \mathrm{d})$ & $55 \cdot 4$ & $22 \cdot 0$ & 81.5 & 30.6 & $<0.001$ & $68 \cdot 7$ & $25 \cdot 4$ & 61.5 & 23.3 & 0.001 & 52.4 & $26 \cdot 2$ & 80.9 & $27 \cdot 2$ & $<0.001$ & 57.9 & $25 \cdot 8$ & $75 \cdot 5$ & 31.4 & $<0.001$ \\
\hline Dietary fibre $(\mathrm{g} / \mathrm{d}) \dagger$ & & & & & & & & & & & & & & & & & & & & \\
\hline Mean & 13 & & & & $<0.001$ & $9 \cdot 1$ & & 16 & & $<0.001$ & 10 & & 13 & & 0.002 & 11 & & 11 & & 0.825 \\
\hline $\mathrm{SE}$ & 0. & & 0 & & & 0.5 & & 0.5 & & & 0. & & 0. & & & 0 & & 0 & & \\
\hline Vitamin C $(\mathrm{mg} / \mathrm{d}) \dagger$ & & & & & & & & & & & & & & & & & & & & \\
\hline Mean & 69 & & 83 & & $<0.001$ & 101. & & 58 & & $<0.001$ & 74 & & 75 & & 0.504 & 56 & & 96 & & $<0.001$ \\
\hline $\mathrm{SE}$ & 2 . & & 2 & & & $2 \cdot 2$ & & $2 \cdot 2$ & & & 2. & & 2 & & & 2 & & 2 & & \\
\hline$M g(m g / d) \dagger$ & & & & & & & & & & & & & & & & & & & & \\
\hline Mean & 325 & & 30 & & $<0.001$ & 292. & & 362 & & $<0.001$ & 230 & & 34 & & $<0.001$ & 30 & & 31 & & $<0.001$ \\
\hline $\mathrm{SE}$ & 5 & & 5 & & & $5 \cdot 1$ & & $5 \cdot 1$ & & & 5 & & 5 & & & 5 & & 5 & & \\
\hline $\mathrm{Zn}(\mathrm{mg} / \mathrm{d}) \dagger$ & & & & & & & & & & & & & & & & & & & & \\
\hline Mean & 11 & & 12 & & $<0.001$ & $12 \cdot 6$ & & 12 & & $<0.001$ & 11 & & 13 & & $<0.001$ & 12 & & 12 & & 0.027 \\
\hline $\mathrm{SE}$ & 0. & & 0 & & & 0.1 & & 0.1 & & & 0. & & 0. & & & 0 & & 0 & & \\
\hline $\mathrm{Se}(\mu \mathrm{g} / \mathrm{d}) \dagger$ & & & & & & & & & & & & & & & & & & & & \\
\hline Mean & 29 & & 42 & & $<0.001$ & $32 \cdot 6$ & & 36 . & & $<0.001$ & 31 & & 41 & & $<0.001$ & 35 & & 37 & & $<0.001$ \\
\hline SE & 0. & & 0 & & & 0.5 & & 0.5 & & & 0. & & 0. & & & 0 & & 0 & & \\
\hline Alcohol intake $(\mathrm{g} / \mathrm{d}) \dagger$ & & & & & & & & & & & & & & & & & & & & \\
\hline Mean & 25 & & 8 & & $<0.001$ & $7 \cdot 2$ & & 15 & & $<0.001$ & 11 & & 24 & & 0.009 & & & 38 & & $<0.001$ \\
\hline SE & 2. & & 2 & & & 2.9 & & 2.9 & & & 3. & & 3. & & & 2 & & 3 & & \\
\hline Beer intake $(\mathrm{g} / \mathrm{d}) \dagger$ & & & & & & & & & & & & & & & & & & & & \\
\hline Mean & 17 & & 26 & & 0.097 & 44.6 & & 12 . & & $<0.001$ & 27 & & 28 & & 0.719 & 5 & & 87 & & $<0.001$ \\
\hline SE & 5. & & 5 & & & 5.4 & & 5.4 & & & 5. & & 5. & & & 5 & 2 & 5 & & \\
\hline Wine intake $(g / d) \dagger$ & & & & & & & & & & & & & & & & & & & & \\
\hline Mean & 3. & & 3 & & 0.994 & 8.9 & & 0.1 & & 0.010 & 12 & & 0. & & $<0.001$ & 0 & & 12 & & $<0.001$ \\
\hline SE & 2 . & & 2 & & & 2.0 & & $2 \cdot 0$ & & & 2. & & 2. & & & 2 & 0 & 2 & & \\
\hline
\end{tabular}

MET, metabolic equivalents.

${ }^{*} P$ values for linear trend for continuous variables (general linear model) and for categorical variables $\left(\chi^{2}\right.$ test for trend).

† Mean values adjusted for sex, age and total energy intake. 
The sample characteristics and dietary intakes of the study participants across the quartiles of the four dietary patterns are presented in Table 2 . Those in the top quartile of all dietary patterns except the Traditional pattern were younger. Those in the top quartile of the Western and High-wheat patterns were more likely to be women, while the opposite was found in the other two patterns. A significant increasing trend of smoking status was observed in the Traditional and Hedonic patterns. A larger BMI was found with the increasing quartiles of the Traditional pattern, while the opposite trend was found in the Hedonic pattern. The physical activity level was increasing across the higher quartile of the Hedonic pattern, while the opposite trend was identified in the Western and High-wheat patterns.

There was a significant positive association between the total energy intake and the Western, Traditional and Hedonic patterns, while a negative association existed in the Highwheat pattern ( $P$ for trend $<0 \cdot 001$ ). The intake of protein, fat, $\mathrm{Zn}$ and Se increased, while that of carbohydrate, dietary fibre and $\mathrm{Mg}$ decreased except for vitamin $\mathrm{C}$, across the quartiles of the Western pattern. All the nutrient intakes mentioned above increased except fat, vitamin $\mathrm{C}$ and $\mathrm{Zn}$ across the quartiles of the High-wheat pattern. There was a clear positive association between all the nutrient intakes except vitamin $\mathrm{C}$ and the Traditional pattern. In the Hedonic pattern, all intakes of macronutrients and micronutrients mentioned, except dietary fibre, increased across the quartiles ( $P$ for trend $<0 \cdot 001$ ). Alcohol intake decreased across the quartiles of the Western pattern, while it increased across the quartiles of the Hedonic pattern. Intakes of beer and wine increased across the quartiles of the Hedonic pattern also ( $P$ for trend $<0.001$ ).

The associations between the dietary patterns and insulin resistance by using multivariate logistic regression are shown in Table 3. No significant interactions were found between dietary pattern and sex (data not shown). Therefore, sex was included as one of the covariates. The Western pattern was positively associated with insulin resistance both in the crude model ( $P$ for trend $<0.001$ ) and in the model adjusted for potential confounders ( $P$ for trend=0.009). Compared with the lowest quartile of the Western pattern, the highest quartile had higher odds of insulin resistance (adjusted OR 1.89, 95\% CI 1.12, 3.19). The High-wheat pattern was also positively associated with insulin resistance in the crude model ( $P$ for trend $=0.018$ ) and in the model adjusted for all the confounders except BMI ( $P$ for trend=0.033); however, the significance disappeared after plenary adjustment. No relationship was seen with the Traditional pattern. A significant negative association was found between the Hedonic pattern and insulin resistance $(P$ for trend $=0.001$ for the crude model and 0.013 for the further adjusted model). BMI attenuated this association slightly after additional adjustment but remained significant ( $P$ for trend $=0.035$ for plenary adjustment). There was a $42 \%$ decrease in the odds after adjustment for all covariates in the highest quartile of the Hedonic pattern, compared with the lowest quartile (adjusted OR 0.58, 95\% CI $0 \cdot 34,0 \cdot 99)$.

Table 3. Multivariate models adjusted for insulin resistance across the quartile ( $Q$ ) categories of the dietary patterns in Jiangsu Province, China (Odds ratios and $95 \%$ confidence intervals)

\begin{tabular}{|c|c|c|c|c|c|c|}
\hline & \multicolumn{2}{|c|}{ Model I* } & \multicolumn{2}{|c|}{ Model II† } & \multicolumn{2}{|c|}{ Model III } \\
\hline & OR & $95 \% \mathrm{Cl}$ & OR & $95 \% \mathrm{Cl}$ & OR & $95 \% \mathrm{Cl}$ \\
\hline \multicolumn{7}{|l|}{ Western } \\
\hline Q1 & 1.00 & & 1.00 & & 1.00 & \\
\hline Q2 & 1.26 & $0.82,1.93$ & 1.33 & $0.83,2.14$ & 1.25 & $0.76,2.06$ \\
\hline Q3 & 2.04 & $1.36,3.08$ & 2.09 & $1.29,3.41$ & 1.90 & $1 \cdot 13,3 \cdot 21$ \\
\hline Q4 & 2.03 & $1.35,3.06$ & 2.09 & $1.28,3.43$ & 1.89 & $1 \cdot 12,3.19$ \\
\hline$P$ for trend & \multicolumn{2}{|c|}{$<0.001$} & \multicolumn{2}{|r|}{0.001} & \multicolumn{2}{|r|}{0.009} \\
\hline \multicolumn{7}{|l|}{ High-wheat } \\
\hline Q1 & 1.00 & & 1.00 & & 1.00 & \\
\hline Q2 & 1.83 & $1 \cdot 21,2 \cdot 77$ & 1.66 & $1.04,2.65$ & 1.49 & $0.91,2.47$ \\
\hline Q3 & 1.88 & $1.24,2 \cdot 84$ & 1.67 & $1.03,2.72$ & 1.54 & $0.92,2.59$ \\
\hline Q4 & 1.73 & $1.14,2.62$ & 1.68 & $1.07,2.63$ & 1.48 & $0.91,2.39$ \\
\hline$P$ for trend & \multicolumn{2}{|r|}{0.018} & \multicolumn{2}{|r|}{0.033} & \multicolumn{2}{|r|}{0.127} \\
\hline \multicolumn{7}{|l|}{ Traditional } \\
\hline Q1 & 1.00 & & 1.00 & & 1.00 & \\
\hline Q2 & 1.03 & $0.69,1.53$ & 0.99 & $0.63,1.55$ & 0.87 & $0.54,1.42$ \\
\hline Q3 & 1.21 & $0.82,1.79$ & $1 \cdot 26$ & $0.80,1.98$ & 1.07 & $0.66,1.73$ \\
\hline Q4 & $1 \cdot 13$ & $0.76,1.68$ & 1.19 & $0.73,1.94$ & 1.05 & $0.62,1.76$ \\
\hline$P$ for trend & \multirow{2}{*}{\multicolumn{2}{|c|}{0.404}} & \multicolumn{2}{|r|}{0.329} & \multicolumn{2}{|r|}{0.668} \\
\hline \multicolumn{5}{|l|}{ Hedonic } & & \\
\hline Q1 & 1.00 & & 1.00 & & 1.00 & \\
\hline Q2 & 0.56 & $0.38,0.82$ & 0.53 & $0.34,0.81$ & 0.60 & $0.38,0.95$ \\
\hline Q3 & 0.55 & $0.37,0.81$ & 0.55 & $0.35,0.86$ & 0.57 & $0.35,0.92$ \\
\hline Q4 & 0.51 & $0.33,0.77$ & 0.54 & $0.33,0.88$ & 0.58 & $0.34,0.99$ \\
\hline$P$ for trend & \multicolumn{2}{|r|}{0.001} & \multicolumn{2}{|r|}{0.013} & \multicolumn{2}{|r|}{0.035} \\
\hline
\end{tabular}

* Adjusted for sex and age ( $<40,40-49,50-59$ or $\geq 60$ years).

†Further adjusted for income (low, medium or high), energy intake (kJ/d), physical activity (metabolic equivalents $\times \mathrm{h} /$ week) and smoking status (yes/no).

$\ddagger$ Additionally adjusted for BMI $\left(<18 \cdot 5,18 \cdot 5-23 \cdot 9,24-27 \cdot 9\right.$ or $\left.>28 \mathrm{~kg} / \mathrm{m}^{2}\right)$. 
In order to identify the association between the dietary patterns and insulin resistance more clearly, we performed further analyses. A significant negative correlation between logarithmically transformed HOMA-IR and the Hedonic pattern scores as continuous variables (Pearson's $r-0 \cdot 161$, $P<0 \cdot 001)$ and a significant positive correlation between transformed HOMA-IR and the Western pattern (Pearson's $r$ 0.142, $P<0.001)$ were found (data not shown).

As shown in Table 4, we observed that HOMA-IR levels as a continuous variable increased across the quartiles of the Western pattern and decreased across the quartiles of the Hedonic pattern. We also found that the shifts of HOMA-IR levels were mainly influenced by the significant change in fasting insulin level instead of glucose level. Finally, in an additional sensitivity analysis, we excluded those subjects with other known chronic diseases (hypertension, myocardial infarction or stroke); the results were consistent (data not shown).

\section{Discussion}

We derived four dietary patterns in our population by using factor analysis: the Western, High-wheat, Traditional and Hedonic pattern. Further analysis indicated that the Western pattern was associated with greater odds of insulin resistance. In contrast, the Hedonic pattern was negatively associated with insulin resistance in Chinese adults. Both associations were independent of sex, age, BMI, income, energy intake, physical activity and smoking status. quartiles of the four dietary patterns

(Medians and interquartile ranges (IQR))
A dietary pattern approach has been used to identify the association between holistic diet exposure and diabetes in China $^{(11,12)}$. In the present study, the positive association between the Western pattern and insulin resistance is similar to previous studies ${ }^{(10,11)}$

Considering the background of an alarming increase in the prevalence of diabetes ${ }^{(25)}$ and other non-communicable diseases, and nutrition transition ${ }^{(26,27)}$ happening in China, we suggest avoiding such an eating pattern with high intakes of energy and fat and low intake of dietary fibre among the population. However, the food groups of the Western pattern identified in the present study cannot be comparable directly with others, especially those undertaken in Western countries $^{(10)}$. Pork, organ meat and soyabean milk are characterised in the 'Western' pattern of our population, while processed meats, beef and butter/cheese are featured in the 'Western' pattern of Western populations.

The negative association between the Hedonic pattern and insulin resistance is a new and important finding in the present study of the Chinese population. Such an association was independent of sex, age, BMI, income, energy intake, physical activity and smoking status. The present finding was somewhat consistent with others done in different populations ${ }^{(4,5,28-30)}$. The results from a meta-analysis in 2005 showed that a U-shaped relationship between alcohol consumption and type 2 diabetes was found. Moderate alcohol consumption was protective against type 2 diabetes in men and women $^{(31)}$. Another meta-analysis study completed in 2009

Table 4. Fasting glucose, insulin, homeostasis model assessment for insulin resistance (HOMA-IR) levels and insulin resistance across the

\begin{tabular}{|c|c|c|c|c|c|c|c|c|}
\hline & \multirow[b]{2}{*}{ Participants $(n)$} & \multicolumn{2}{|c|}{ Glucose $(\mathrm{mmol} / \mathrm{l})^{\star}$} & \multicolumn{2}{|c|}{ Insulin $(\mathrm{pmol} / \mathrm{l})^{*}$} & \multicolumn{2}{|c|}{ HOMA-IR (units)* } & \multirow[b]{2}{*}{ Insulin resistance (\%) } \\
\hline & & Median & IQR & Median & IQR & Median & IQR & \\
\hline \multicolumn{9}{|l|}{ Western } \\
\hline Q1 & 267 & $5 \cdot 1$ & $4.7-5 \cdot 4$ & $23 \cdot 1$ & $15 \cdot 1-39 \cdot 6$ & 0.9 & $0.5-1.5$ & $17 \cdot 6$ \\
\hline Q2 & 268 & $5 \cdot 0$ & $4 \cdot 6-5 \cdot 4$ & 30.9 & $20 \cdot 1-43 \cdot 7$ & $1 \cdot 1$ & $0.7-1.7$ & $21 \cdot 6$ \\
\hline Q3 & 268 & $5 \cdot 0$ & $4 \cdot 6-5 \cdot 4$ & 32.5 & $19 \cdot 8-49 \cdot 0$ & 1.3 & $0.7-2 \cdot 0$ & $30 \cdot 6$ \\
\hline Q4 & 267 & $5 \cdot 0$ & $4.5-5 \cdot 6$ & 32.5 & $21 \cdot 4-51 \cdot 1$ & $1 \cdot 2$ & $0.8-2.0$ & $30 \cdot 3$ \\
\hline$P$ for trend $\dagger$ & & \multicolumn{2}{|c|}{0.604} & \multicolumn{2}{|c|}{0.003} & \multicolumn{2}{|c|}{0.003} & $<0.001$ \\
\hline \multicolumn{2}{|l|}{ High-wheat } & & & & & & & \\
\hline Q1 & 267 & 4.9 & $4 \cdot 6-5 \cdot 4$ & 24.4 & $17 \cdot 1-39 \cdot 3$ & 0.9 & $0.6-1.4$ & $17 \cdot 2$ \\
\hline Q2 & 268 & $5 \cdot 0$ & $4 \cdot 6-5 \cdot 6$ & 31.5 & $19 \cdot 8-49 \cdot 0$ & 1.2 & $0.7-1.9$ & $27 \cdot 6$ \\
\hline Q3 & 268 & $5 \cdot 1$ & $4 \cdot 6-5 \cdot 7$ & 32.3 & $20 \cdot 0-46.5$ & $1 \cdot 2$ & $0.8-1.9$ & 28.4 \\
\hline Q4 & 267 & $5 \cdot 0$ & $4 \cdot 6-5 \cdot 4$ & $30 \cdot 7$ & $19 \cdot 6-49 \cdot 1$ & 1.2 & $0.7-1.9$ & $27 \cdot 0$ \\
\hline$P$ for trend $\dagger$ & & \multicolumn{2}{|c|}{0.001} & \multicolumn{2}{|c|}{0.392} & \multicolumn{2}{|c|}{0.235} & 0.012 \\
\hline \multicolumn{2}{|l|}{ Traditional } & & & & & & & \\
\hline Q1 & 267 & 4.9 & $4.5-5.5$ & $31 \cdot 1$ & $19 \cdot 2-48 \cdot 0$ & $1 \cdot 1$ & $0.7-1.8$ & 24.0 \\
\hline Q2 & 268 & $5 \cdot 0$ & $4 \cdot 6-5 \cdot 4$ & $28 \cdot 7$ & $18 \cdot 5-43 \cdot 7$ & $1 \cdot 1$ & $0.7-1.7$ & 23.9 \\
\hline Q3 & 268 & $5 \cdot 0$ & $4 \cdot 6-5 \cdot 4$ & 29.8 & $19 \cdot 1-47 \cdot 0$ & $1 \cdot 1$ & $0.7-1.8$ & $26 \cdot 9$ \\
\hline Q4 & 267 & $5 \cdot 1$ & $4 \cdot 7-5 \cdot 7$ & $29 \cdot 3$ & $19 \cdot 3-45 \cdot 0$ & $1 \cdot 1$ & $0.7-1 \cdot 8$ & $25 \cdot 5$ \\
\hline$P$ for trend $t$ & & \multicolumn{2}{|c|}{0.004} & \multicolumn{2}{|c|}{0.933} & \multicolumn{2}{|c|}{0.731} & 0.527 \\
\hline \multicolumn{2}{|l|}{ Hedonic } & & & & & & & \\
\hline Q1 & 267 & $5 \cdot 1$ & $4 \cdot 6-5 \cdot 5$ & $37 \cdot 0$ & $23 \cdot 0-55 \cdot 0$ & 1.4 & $0.8-2.2$ & 34.5 \\
\hline Q2 & 268 & $5 \cdot 0$ & $4 \cdot 6-5 \cdot 5$ & 30.9 & $20 \cdot 5-44.6$ & 1.2 & $0.8-1.8$ & $23 \cdot 1$ \\
\hline Q3 & 268 & $5 \cdot 0$ & $4 \cdot 6-5 \cdot 4$ & $28 \cdot 2$ & $18 \cdot 0-44 \cdot 4$ & 1.0 & $0.7-1.7$ & 22.4 \\
\hline Q4 & 267 & $5 \cdot 0$ & $4 \cdot 6-5 \cdot 4$ & 24.4 & $15 \cdot 1-38 \cdot 8$ & 0.9 & $0.6-1.6$ & $20 \cdot 2$ \\
\hline$P$ for trend $\dagger$ & & \multicolumn{2}{|c|}{0.021} & \multicolumn{2}{|c|}{0.015} & \multicolumn{2}{|c|}{0.051} & $<0.001$ \\
\hline
\end{tabular}

* Log-transformed when testing for linear trends.

† Adjusted for sex and age ( $<40,40-49,50-59$, or $\geq 60$ years), income (low, medium or high), energy intake (kJ/d), physical activity (metabolic equivalents $\times \mathrm{h} /$ week), smoking status (yes/no) and BMI $\left(<18.5,18.5-23.9,24-27.9 \mathrm{or}>28 \mathrm{~kg} / \mathrm{m}^{2}\right)$. 
also confirmed these findings ${ }^{(32)}$. Such findings are supported in individual studies from different populations ${ }^{(29,30)}$.

Available epidemiological evidence showed that the negative association between the Hedonic pattern and insulin resistance could be attributable to the pattern's healthy components, which may include alcohol in a moderate amount $^{(31,32)}$, fresh vegetables ${ }^{(2)}$, tubers ${ }^{(33)}$ and some micronutrients such as vitamin $\mathrm{C}^{(34)}, \mathrm{Mg}^{(35,36)}, \mathrm{Zn}^{(37)}$ and $\mathrm{Se}^{(38)}$. Contribution of these components was supported by the present results. Reduced insulin resistance may be due to their synergistic effect. As mentioned above, moderate alcohol consumption was independently associated with insulin resistance or diabetes. The average intake of alcohol in the present study population was actually low $(15.5 \mathrm{~g} / \mathrm{d}$ for alcohol, $27.6 \mathrm{~g} / \mathrm{d}$ for beer and $3.0 \mathrm{~g} / \mathrm{d}$ for wine). Even the mean intake of alcohol in the highest quartile of the Hedonic pattern was $38.5 \mathrm{~g} / \mathrm{d}$, which was still lower than the upper limit of moderate alcohol consumption ${ }^{(31,32)}$. Therefore, the present findings correspond to the left side and bottom of the U-shaped relationship found in previous results ${ }^{(31,32)}$

Research focusing on the health effects of alcohol drinking has increasingly emerged in recent years, but little can be found about its protective effects among the Chinese population. It remains unclear that whether the Hedonic pattern has a causal protective mechanism on insulin resistance; therefore, clinical and public health recommendations from such findings may be too early. However, advice on reducing heavy alcohol drinking is always appropriate. The Dietary Guidelines for Americans and Chinese consistently suggest 'if alcohol is consumed, it should be consumed in moderation'.

As we mentioned previously, a dietary pattern approach overcomes the defects of an individual food analysis, which is mainly limited by biological interactions. Normally, alcohol is not consumed separately in China but is consumed as part of specified dietary patterns. Therefore, the present study adds new evidence to previous research findings that the Hedonic dietary pattern is inversely associated with insulin resistance.

The strengths of the present study include the use of a validated semi-quantitative FFQ by a face-to-face interview, which ensured that the data we collected were accurate. Further, the findings are reliable because we adjusted for potential known confounders in the models. Moreover, as we excluded the subjects with known diabetes, it is unlikely that the results are confounded by diabetic medication.

However, limitations also need to be considered in the interpretation of the present findings. The main limitation of the present study is its cross-sectional nature. Reverse causation would be a possibility if health outcomes influence the intake of alcohol rather than the reverse. Although we excluded subjects with other known chronic diseases in sensitivity analysis, such a possibility has not been fully addressed here. The present findings remain to be verified by future prospective studies. Furthermore, we cannot generalise the present findings to all Chinese populations because there are considerable differences in eating habit, food categories and even cooking methods given such a large geographical diversity. Participants in the present study were sampled from six socio-economically and geographically different areas. So these patterns can represent the situation among the population in Jiangsu Province, Eastern China. Even in the same province, due to different study samples, different dietary patterns could be identified ${ }^{(39)}$. However, the cluster of wheat and rice in the opposite direction is consistent in different studies in China. The factor loading for the Highwheat pattern was 0.75 for wheat and -0.68 for rice in the present study, while the factor loading for the 'traditional' pattern was -0.75 for wheat and 0.78 for rice in another study in the province ${ }^{(39)}$.

In conclusion, the present findings indicate that the Western pattern was associated with greater odds of insulin resistance. In contrast, the Hedonic pattern was associated with decreased odds of insulin resistance in Chinese adults. Such associations were independent of sex, age, BMI, income, energy intake, physical activity and smoking status.

\section{Acknowledgements}

The authors are sincerely grateful to the CHNS project initiated by the Carolina Population Center at the University of North Carolina at Chapel Hill and the National Institute of Nutrition and Food Safety at the Chinese Center for Disease Control and Prevention. Also, we thank the entire study team and all participants in the study. This research received no specific grant from any funding agency in the public, commercial or not-for-profit sectors. H. Z. designed and conducted the analysis, interpreted the data, and wrote and edited the manuscript. Z. S. contributed to the design, data analysis and discussion, and reviewed the manuscript. B. Y., Y. D. and G. W. researched the data. X. P. contributed to the data analysis. A. H. contributed to the design and data interpretation, and reviewed the manuscript. The authors declare that they have no conflicts of interest.

\section{References}

1. Lann D \& LeRoith D (2007) Insulin resistance as the underlying cause for the metabolic syndrome. Med Clin North Am 91, 1063-1077.

2. Spence M, McKinley MC \& Hunter SJ (2009) Session 4: CVD, diabetes and cancer diet, insulin resistance and diabetes: the right (pro)portions. Proc Nutr Soc 69, 61-69.

3. Tremblay A \& Gilbert JA (2009) Milk products, insulin resistance syndrome and type 2 diabetes. J Am Coll Nutr 28, Suppl. 1, 91S-102S.

4. Gunji T, Matsuhashi N, Sato H, et al. (2011) Alcohol consumption is inversely correlated with insulin resistance, independent of metabolic syndrome factors and fatty liver diseases. J Clin Gastroenterol 45, 808-813.

5. Flanagan DEH, Moore VM, Godsland IF, et al. (2000) Alcohol consumption and insulin resistance in young adults. Eur J Clin Invest 30, 297-301.

6. Isharwal S, Misra A, Wasir JS, et al. (2009) Diet \& insulin resistance: a review \& Asian Indian perspective. Indian J Med Res 129, 485-499.

7. Hu FB (2002) Dietary pattern analysis: a new direction in nutritional epidemiology. Curr Opin Lipidol 13, 3-9.

8. Newby PK \& Tucker KL (2004) Empirically derived eating patterns using factor or cluster analysis: a review. Nutr Rev 62, 177-203. 
9. Kant AK (2004) Dietary patterns and health outcomes. J Am Diet Assoc 104, 615-635.

10. Esmaillzadeh A, Kimiagar M, Mehrabi Y, et al. (2007) Dietary patterns, insulin resistance, and prevalence of the metabolic syndrome in women. Am J Clin Nutr 85, 910-918.

11. He Y, Ma G, Zhai F, et al. (2009) Dietary patterns and glucose tolerance abnormalities in Chinese adults. Diabetes Care 32, 1972-1976.

12. Villegas R, Yang G, Gao YT, et al. (2010) Dietary patterns are associated with lower incidence of type 2 diabetes in middle-aged women: the Shanghai Women's Health Study. Int J Epidemiol 39, 889-899.

13. Odegaard AO, Koh WP, Butler LM, et al. (2011) Dietary patterns and incident type 2 diabetes in Chinese men and women: The Singapore Chinese Health Study. Diabetes Care 34, 880-885.

14. Wang Y, Mi J, Shan XY, et al. (2007) Is China facing an obesity epidemic and the consequences? The trends in obesity and chronic disease in China. Int J Obes (Lond) 31, 177-188.

15. Popkin BM, Du S, Zhai F, et al. (2010) Cohort Profile: The China Health and Nutrition Survey - monitoring and understanding socio-economic and health change in China, 1989-2011. Int J Epidemiol 39, 1435-1440.

16. Willett W (1998) Nutritional Epidemiology. New York: Oxford University Press.

17. Zhao W, Hasegawa K \& Chen J (2006) The use of foodfrequency questionnaires for various purposes in China. Public Health Nutr 5, 829-833.

18. Li YP, He YN, Zhai FY, et al. (2006) Comparison of assessment of food intakes by using 3 dietary survey methods. Zhonghua Yu Fang Yi Xue Za Zhi 40, 273-280.

19. Yang Y (2005) Chinese Food Composition Table 2004. Beijing: Peking University Medical Press.

20. Matthews DR, Hosker JP, Rudenski AS, et al. (1985) Homeostasis model assessment: insulin resistance and beta-cell function from fasting plasma glucose and insulin concentrations in man. Diabetologia 28, 412-419.

21. Isomaa B, Almgren P, Tuomi T, et al. (2001) Cardiovascular morbidity and mortality associated with the metabolic syndrome. Diabetes Care 24, 683-689.

22. Kalish GM (2003) Association of endogenous sex hormones and insulin resistance among postmenopausal women: results from the postmenopausal estrogen/progestin intervention trial. J Clin Endocrinol Metab 88, 1646-1652.

23. Ainsworth BE, Haskell WL, Whitt MC, et al. (2000) Compendium of physical activities: an update of activity codes and MET intensities. Med Sci Sports Exerc 32, S498-S504.

24. Zuo H, Shi Z, Yuan B, et al. (2012) Interaction between physical activity and sleep duration in relation to insulin resistance among non-diabetic Chinese adults. BMC Public Health 12, 247.
25. Yang W, Lu J, Weng J, et al. (2010) Prevalence of diabetes among men and women in China. $N$ Engl J Med 362, 1090-1101.

26. Popkin BM \& Du S (2003) Dynamics of the nutrition transition toward the animal foods sector in China and its implications: a worried perspective. J Nutr 133, 3898S-3906S.

27. Zhai F, Wang H, Du S, et al. (2009) Prospective study on nutrition transition in China. Nutr Rev 67, S56-S61.

28. Imamura F, Lichtenstein AH, Dallal GE, et al. (2009) Confounding by dietary patterns of the inverse association between alcohol consumption and type 2 diabetes risk. Am J Epidemiol 170, 37-45.

29. Sato KK, Hayashi T, Harita N, et al. (2012) Relationship between drinking patterns and the risk of type 2 diabetes: the Kansai Healthcare Study. I Epidemiol Community Health 66, 507-511.

30. Athyros VG, Liberopoulos EN, Mikhailidis DP, et al. (2007) Association of drinking pattern and alcohol beverage type with the prevalence of metabolic syndrome, diabetes, coronary heart disease, stroke, and peripheral arterial disease in a Mediterranean cohort. Angiology 58, 689-697.

31. Koppes LL, Dekker JM, Hendriks HF, et al. (2005) Moderate alcohol consumption lowers the risk of type 2 diabetes: a meta-analysis of prospective observational studies. Diabetes Care 28, 719-725.

32. Baliunas DO, Taylor BJ, Irving $\mathrm{H}$, et al. (2009) Alcohol as a risk factor for type 2 diabetes: a systematic review and meta-analysis. Diabetes Care 32, 2123-2132.

33. Oki N, Nonaka S \& Ozaki S (2011) The effects of an arabinogalactan-protein from the white-skinned sweet potato (Ipomoea batatas L.) on blood glucose in spontaneous diabetic mice. Biosci Biotechnol Biochem 75, 596-598.

34. Hercberg S, Czernichow S \& Galan P (2009) Vitamin C concentration and type 2 diabetes mellitus. Arch Intern Med 169, 634 .

35. Mooren FC, Kruger K, Volker K, et al. (2011) Oral magnesium supplementation reduces insulin resistance in non-diabetic subjects - a double-blind, placebo-controlled, randomized trial. Diabetes Obes Metab 13, 281-284.

36. Guerrero-Romero F, Tamez-Perez HE, Gonzalez-Gonzalez G, et al. (2004) Oral magnesium supplementation improves insulin sensitivity in non-diabetic subjects with insulin resistance. A double-blind placebo-controlled randomized trial. Diabetes Metab 30, 253-258.

37. Kelishadi R, Hashemipour M, Adeli K, et al. (2010) Effect of zinc supplementation on markers of insulin resistance, oxidative stress, and inflammation among prepubescent children with metabolic syndrome. Metab Syndr Relat Disord 8, 505-510.

38. Mueller AS, Mueller K, Wolf NM, et al. (2009) Selenium and diabetes: an enigma? Free Radical Res 43, 1029-1059.

39. Shi Z, Hu X, Yuan B, et al. (2008) Vegetable-rich food pattern is related to obesity in China. Int J Obes 32, 975-984. 